\title{
Unsupervised Learning of Representations from Solar Energy Data
}

\author{
Nicola Piovesan, Paolo Dini \\ CTTC/CERCA, Av. Carl Friedrich Gauss, 7, 08860, Castelldefels, Barcelona, Spain \\ \{npiovesan, pdini\}@cttc.es
}

\begin{abstract}
In this paper, we propose an unsupervised method to learn hidden features of the solar energy generation from a PV system that may give a more accurate characterization of the process. In a first step, solar radiation data is converted into instantaneous solar power through a detailed source model. Then, two different approaches, namely PCA and autoencoder, are used to extract meaningful features from the traces of the solar energy generation. We interpret the latent variables characterizing the solar energy generation process by analyzing the similarities of 67 cities in Europe, North-Africa and Middle-East through an agglomerative hierarchical clustering algorithm. This analysis provides also a comparison between the feature extraction capabilities of the PCA and the autoencoder.
\end{abstract}

Index Terms-Energy sustainability, neural networks, PCA, solar energy, unsupervised learning

\section{INTRODUCTION}

In recent years, several articles have shown that the energy consumption of ICT may represent a problem in terms of sustainability. In a report of 2013, the Digital Power group [1] has calculated that $10 \%$ of the worldwide electricity generation is due to ICT, which is exceeded by more than $50 \%$ by that of the avionic industry. A forecast for 2030 estimates that $51 \%$ of the electricity consumption and $23 \%$ of the carbon footprint by human activity will be due to ICT [2]. This trend is mainly driven by new infrastructure deployments to provide mobile services.

The introduction of renewable energy sources is today one of the most promising approaches to reduce the greenhouse gases emissions due to the human activities. In particular, the reduced cost of the harvesting devices, e.g., Photovoltaic (PV) systems, makes solar energy one of the most interesting sources among the renewables.

Several works in the literature [3], [4], [5] discuss the integration of solar energy sources into communication networks as a way to reduce their energy consumption and conclude that such an integration is feasible only with the introduction of an intelligent control system able to manage the intermittent and erratic energy budget from renewable sources.

One of the key factor determining the performance of a PV system and its potential application to supply, e.g. communication network devices, is the solar energy arriving at the surface of the Earth. The exploitation of the solar energy resource is determined by the knowledge of geographical variability and time dynamics. The geographical analysis of the availability of the primary solar energy resource is then essential to understand the potential implementation of PV systems for future energy supply to a specific industrial sector. The geographical dependency and distributed nature of solar electricity generation impose questions that require specific location-dependent answers. In fact, the harvested energy strictly depends on the seasons of the year and the meteorology of the given location. In [6], it has been estimated that, in the same site, even during summer and in good weather conditions, the harvested energy in the peak irradiation hour can vary up to the $85 \%$. Similarly, considering that the solar radiation intensity and the daylight duration vary significantly across the months [6], seasons have a strong impact on the amount of the harvested energy income. Normally, this geographical analysis is performed using solar maps, which provide easy-understandable information. The total annual solar electricity generation from a PV system is used to characterize national and regional differences [7]. In this paper, we are interested in learning hidden features of the solar energy generation that may give a more accurate characterization of the process beyond that usual metric.

Recently, representation learning has received significant attention as a highly effective alternative to conventional feature sets handcrafted by a domain expert [8]. These techniques have been shown to be superior to feature engineering for a plethora of tasks, including speech recognition, music transcription, audio and video recognition [9]. However, these methods suffer the big issue of the interpretability of their results [8]. In this work, we use unsupervised representation learning methods to extract automatically time-dependent features from solar electricity generation data and provide a meaningful interpretation of the results achieved. Although machine learning methods have been used in renewable energy modeling, they have been adopted mainly to forecast the next energy arrivals in a given location [10]. Instead, to the best of our knowledge, they have not been used for analyzing geographical differences in solar electricity generation. 
In this paper, we state the geographical analysis of the availability of the solar energy generation from a $\mathrm{PV}$ system as an unsupervised learning problem due to the lack of ground truth for the considered scenario. In detail, raw data from a real database describing the solar irradiance on a plane surface in 67 different cities are processed to obtain estimations of the instantaneous solar energy generation. We introduce an astronomical model that allows estimating the actual solar electricity generation by a PV system. Then, an electrical model of the PV module and of the DC/DC power processor are used to provide the final estimation. Feature extraction is performed on the solar electricity generation data using two different approaches: Principal Component Analysis (PCA) and Under-complete AutoEncoder (UAE). PCA is a non-parametric technique that learns a linear transformation of the input data, whereas UAE is a specific artificial neural network architecture that learns the parameters of an encoder and a decoder function minimizing the reconstruction error of the input space. Then, we interpret the latent variables characterizing the solar energy generation process by analyzing the similarities of the different cities with an agglomerative hierarchical clustering algorithm. This analysis provides also a comparison between the feature extraction capabilities of PCA and UAE.

The paper is organized as follows. In Section II we provide a description of the solar irradiance dataset. In Section III we describe the system model used to calculate the solar electricity from a PV system; whereas in Section IV we introduce the features extraction algorithms. Section V discusses the classification performed by the agglomerative hierarchical clustering algorithm and gives an interpretation of the extracted features from the solar energy generation data. Finally, in Section VI we draw our conclusions.

\section{DATASET}

The solar irradiance dataset considered in this work [11], contains hourly solar irradiance values, in $\mathrm{W} / \mathrm{m}^{2}$, collected from February 1st, 2004 to December 31st, 2006. In particular, we consider 67 different cities, located in Europe, Middle East, and North Africa.

The solar electricity generation is estimated using the model described in Section III. In particular, the model parameters are set considering a Panasonic N235B PV module, which has single cell efficiencies of about $21 \%$, delivering about $186 \mathrm{~W} / \mathrm{m}^{2}$. Each module is composed of an array of $16 \times 16$ solar cells (i.e., a surface of $4.48 \mathrm{~m}^{2}$ ). We consider this particular dimension since it represents a realistic size for supplying renewable powered small base stations [12].

We define $\mathcal{D}=\left\{\mathcal{D}^{y_{1}}, \ldots, \mathcal{D}^{y_{K}}\right\}$ as the dataset containing the traces of generated energy, with $K=67$. In particular, for each city $y$, we have a set $\mathcal{D}^{y}=$

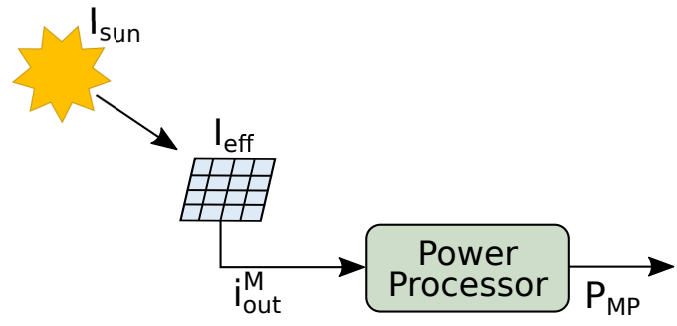

Fig. 1. Source model used to characterize the solar energy generation process.

$\left\{\mathbf{e}_{1}, \ldots, \mathbf{e}_{n}\right\}$ containing $n$ daily traces of the generated energy $\mathbf{e}_{i}, i \in\{1, \ldots, n\}$. The vector $\mathbf{e}_{i}$ has 24 elements, each one containing the amount of energy generated in the respective hour of the day. Since our dataset contains 35 months of measurements, $n=1069$.

\section{SySTEM MODEL}

In this section, we describe the source model used to characterize the electricity generated by a PV panel. The key building blocks of the model are illustrated in Fig. 1 and they are the solar source, the photovoltaic panel and the DC/DC power processor.

\section{A. Astronomical model}

We define the effective solar irradiance that hits a photovoltaic panel as $I_{\text {eff }}$. This term depends on several factors, e.g., the inclination of the panel, the geographical location, the hour of the day and the day of the year.

In order to translate the solar irradiance, $I_{\text {sun }}$, into effective solar irradiance, $I_{\text {eff }}$, we consider the following astronomical model. According to [13], the effective solar irradiance, $I_{\text {eff }}$ is proportional to $\cos \Theta$, where $\Theta \in\left[0,90^{\circ}\right]$ is the angle between the sunlight and the normal to the solar module surface. It can be computed as a function of time $t$ by $I_{\text {eff }}(t, N)=$ $I_{\text {sun }}(t, N) \max (0, \cos \Theta(t, N))$, where $N$ is the day number in a year (i.e., $N=1$ for January 1st and $N=365$ for December 31st).

The value of $\cos \Theta$ is calculated as:

$$
\begin{aligned}
& \cos \Theta(t, N)=\sin \gamma(N) \cdot \sin L a \cdot \cos \beta- \\
& \quad-\sin \gamma(N) \cdot \cos L a \cdot \sin \beta \cdot \cos \alpha+ \\
& \quad+\cos \gamma(N) \cdot \cos L a \cdot \cos \beta \cdot \cos \omega(t, N)+ \\
& \quad+\cos \gamma(N) \cdot \sin L a \cdot \sin \beta \cdot \cos \alpha \cdot \cos \omega(t, N)+ \\
& \quad+\cos \gamma(N) \cdot \sin \beta \cdot \sin \alpha \cdot \sin \omega(t, N)
\end{aligned}
$$

where:

- $L a \in\left[0,90^{\circ}\right]$ is the location latitude;

- Lo is the location longitude;

- $\gamma$ is the declination angle;

- $\omega(t, N) \in\left[0,360^{\circ}\right]$ is the hour angle;

- $\beta$ is the inclination of the solar panel towards the sun on the horizon; 
- $\alpha$ is the azimuthal displacement, which takes values different from zero if the normal to the plane of the solar panel is not aligned with the plane of the corresponding meridian i.e., the solar panel faces East $(\alpha<0)$ or West $(\alpha>0)$.

The declination angle $\gamma$ is due to the elliptic orbit of the Earth around the sun and the fact the Earth is tilted on itself at an angle of $23.45^{\circ}$. It is defined as the angular distance North or South of the Earth's equator and it can be calculated as:

$$
\gamma(N) \simeq \sin ^{-1}\left[\sin \left(23.45^{\circ}\right) \sin (D(N))\right]
$$

where $D(N)=360(N-81) / 365^{\circ}$.

The hour angle $\omega$ is defined as the azimuth's angle of the sun's rays due to the Earth's rotation and it can be calculated as:

$$
\omega(t, N)=15(\operatorname{AST}(t, N)-12)^{\circ}
$$

where $\operatorname{AST}(t, N) \in[0,24]$ hour is the apparent solar time. We can calculate it as:

$$
A S T(t, N)=t^{\prime}+\Delta t+\mathrm{ET}(N)
$$

where $t^{\prime}$ is the local standard time adjusted to account for the daylight saving time. $\Delta t$ is the time displacement between the selected time zone and the time at the reference Greenwich meridian. It is computed as $\Delta t=(L o-G M A) / 15^{\circ}$ where $G M A=U T C_{\text {off }} \times 15^{\circ}$ is the Greenwich meridian angle and corresponds to the angle between the Greenwich meridian and the meridian of the selected time zone. $U T C_{\text {off }}$ is the time offset between Greenwich and the time zone whereas 15 is the rotation angle of the Earth per hour. Finally, $E T(N) \simeq[9.87 \sin (2 D(N))-7.53 \cos (D(N))-$ $1.5 \sin (D(N))] / 60$ is known as the equation of time.

\section{B. Solar panel model}

We consider a solar panel composed of $n_{\mathrm{sc}}$ solar cells connected together. A number $n_{\mathrm{p}}$ of them are connected in parallel, whereas $n_{\mathrm{s}}$ are connected in series. Thus, $n_{\mathrm{sc}}=n_{\mathrm{p}} n_{\mathrm{s}}$.

The composition of the I-V curves of the solar cells allows obtaining the I-V curve used to characterize the solar panel. The I-V curve of a solar cell is given by the superposition of the current generated by the solar cell diode in the dark with the current due to the sunlight hitting the cell, defined as light-generated current $i_{l}$. We can approximate this curve as:

$$
i_{\mathrm{out}} \simeq i_{l}-i_{\mathrm{o}}\left[\exp \left(\frac{q v}{n k T}\right)-1\right]
$$

where $q$ is the elementary charge, $v$ is the cell voltage, $k$ is the Boltzmann's constant, $T$ is the temperature in Kelvin degrees, $n \geq 1$ is the diode ideal factor. Finally, $i_{\mathrm{o}}$ is the dark saturation current and corresponds to the diode leakage current when there is not light. It depends on the area and the technology of the solar cell.
We define $i_{\mathrm{sc}}$ as the short circuit current, which corresponds to the maximum current for the cell. We can normalize the effective irradiance, $I_{\text {eff }}$ with respect to the maximum radiation of $1 \mathrm{~kW} / \mathrm{m}^{2}$, obtaining the radiation rate $F(t, N)=0.001 \cdot I_{\text {eff }}(t, N)$. Then, we can compute the light-generated current for a single solar cells as $i_{l}(t, N)=i_{\mathrm{sc}} F(t, N)$ and obtain $i_{\text {out }}(t, N)$ for a single solar cells using equation (5). Finally, the total current generated by the solar module is $i_{\mathrm{out}}^{\mathrm{M}}(t, N)=$ $n_{\mathrm{p}} i_{\text {out }}(t, N)$.

\section{Power processor model}

Every voltage or current source has a maximum power point, at which the average power delivered to its load is maximized. In general, the load of a device does not match the optimal one, required to extract the maximum power from the solar source. To solve this problem, a power processor is used to emulate the optimal load by adjusting the source voltage until the power extracted from it is maximized.

In this paper, in order to account for the DC/DC power processor, we have computed the operating point $\left(i_{\mathrm{out}}^{\mathrm{M}}, v^{\mathrm{M}}\right)$ for which the extracted power $P=i_{\mathrm{out}}^{\mathrm{M}} v^{\mathrm{M}}$ is maximized.

We have obtained the maximum power $P_{\mathrm{MP}}$ as:

$$
P_{\mathrm{MP}}=\eta \max _{v}\left\{i_{\mathrm{out}}^{\mathrm{M}}, v^{\mathrm{M}}\right\}=\eta \cdot n_{\mathrm{p}} n_{\mathrm{s}} \max _{v}\left\{i_{\mathrm{out}} v\right\}
$$

where $i_{\text {out }}$ is given by equation (5) and $\eta \in(0,1)$ is the power processor conversion efficiency.

\section{FEATURE EXTRACTION}

Two different approaches have been used to reduce the dimensionality of the input data and extract meaningful features: PCA and UAE, described in the following sub-sections.

\section{A. Principal Component Analysis (PCA)}

PCA is a non-parametric technique for extracting relevant features from a dataset. The purpose is to reduce the dimensionality of the dataset by finding a new set of variables, smaller than the original, that retains most of the original information. Those new variables are called principal components (PC). They are uncorrelated and they are ordered by the fraction of the total information each retains [14].

Given $n$ observations of the vector $\mathbf{e}=\left(e_{1}, \ldots, e_{24}\right)$, the first PC is computed as:

$$
z_{1} \equiv \mathbf{a}_{1}^{T} \mathbf{e}=\sum_{i=1}^{24} a_{i, 1} e_{i}
$$

where $\mathbf{a}_{1}=\left(a_{1,1}, a_{2,1}, \ldots, a_{24,1}\right)$ is the vector that maximize the variance of $z_{1}$.

In a similar way, the $k^{\text {th }} \mathrm{PC}$ (with $k=1, \ldots, 24$ ) is computed as:

$$
z_{k} \equiv \mathbf{a}_{k}^{T} \mathbf{e}
$$




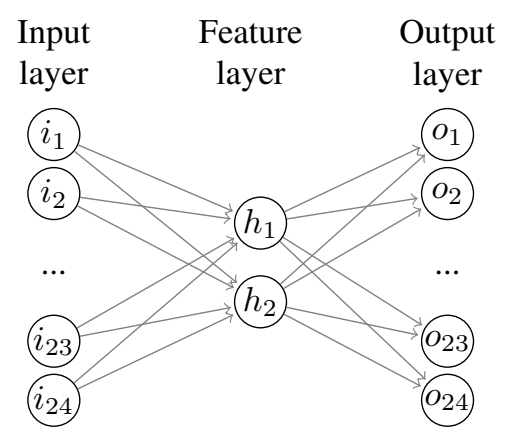

Fig. 2. Autoencoder topology used for extracting features of the solar energy generation of the 67 cities in the dataset.

where the vector $\mathbf{a}_{k}$ is chosen such that the variance of $z_{k}$ is maximum, subject to $\operatorname{cov}\left[z_{k}, z_{l}\right]=0$ for $k>l \geq 1$ and $\mathbf{a}_{k}^{T} \mathbf{a}_{k}=1$.

The generic observation $\mathbf{e}_{i}$ can be written as the sum of its PCs:

$$
\mathbf{e}_{i}=\sum_{k=1}^{24} z_{i, k} \mathbf{a}_{k}
$$

According to the given definitions, it can be easily proved that the first PC retains the greatest amount of variation in the sample, whereas the $k^{\text {th }} \mathrm{PC}, z_{k}$, retains the greatest $k^{\text {th }}$ fraction of the variation in the sample. This fact allows us to approximate each observation by truncating the sum at the first $m<24$ PCs:

$$
\mathbf{e}_{i} \simeq \mathbf{e}_{i}^{m}=\sum_{k=1}^{m} z_{i, k} \mathbf{a}_{k}
$$

The number of considered PCs has been set to $m=2$ since tests performed on the available data shows that the first 2 PCs retains the $94 \%$ of the information (respectively the $79 \%$ and the $15 \%$ for the 1 st and the 2nd PC). Thus, we can associate to each observation $\mathbf{e}_{i}$ a feature vector $\mathbf{h}_{i}^{\text {PCA }}=\left[z_{i, 1}, z_{i, 2}\right]$. In this way, for each $\mathcal{D}^{y}$, we obtain a set of 2-dimensional features $\mathcal{F}_{\mathrm{PCA}}^{y}=\left\{\mathbf{h}_{1}^{\mathrm{PCA}}, \ldots, \mathbf{h}_{n}^{\mathrm{PCA}}\right\}$, which is a compressed representation of the evolution of the solar energy generation in the city $y$.

\section{B. Under-complete Autoencoder (UAE)}

An under-complete autoencoder (UAE) is an artificial neural network used for unsupervised learning of representations from a set of data, for the purpose of dimensionality reduction [15]. It learns to compress data from the input layer into a short code, and then uncompress that code into something that closely matches the original data (output layer). The set of hidden layers with decreasing number of neurons till reaching the central layer is called encoder. Another set of hidden layers from the central layer to the output layer is for the reconstruction of the original data and named decoder.

Figure 2 shows the autoencoder used in this work. The input and output layers are composed of 24 neurons,

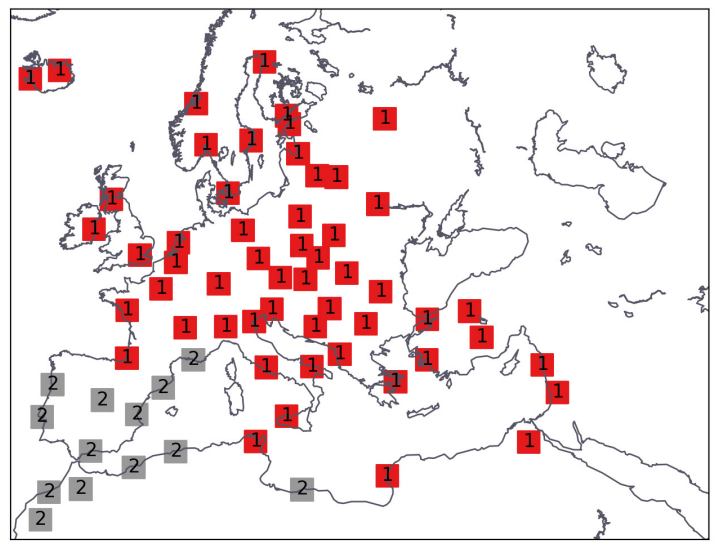

Fig. 3. Clusters obtained by using the PCA approach.

each one representing the amount of energy generated in the corresponding hour of the day. The single hidden layer, named feature layer, is composed of 2 neurons. In this way, the information about the 24-hour solar energy generation trace is coded into a feature of dimension 2 .

We consider the Multilayer Perceptron as basic architecture, consisting of multiple fully connected layers of neurons. The output of a neuron is computed using the following equation:

$$
\text { output }=f\left(\sum_{i}\left(x_{i} \cdot w_{i}+b_{i}\right)\right)
$$

where $x$ is the input of the neuron, $w$ is the weight of the connection to the neuron, $b$ is the bias and $f$ is the activation function. The neurons of the input and feature layers use the ReLU as activation function $\left(f_{a}(x)=\right.$ $\max (0, x))$, whereas the neurons of the output layer use the sigmoid function $\left(f_{b}(x)=1 /\left(1+e^{-x}\right)\right)$.

The dataset $\mathcal{D}$ is split into two portions: the training set $\mathcal{T}$, containing $n_{t}=730$ daily traces and the validation set $\mathcal{V}$ containing the remaining $n-n_{t}$ daily traces. The first set is used for training the autoencoder. During this phase, the backpropagation algorithm iteratively updates the weights of the connections between neurons to minimize the reconstruction loss (training loss). At the same time, the autoencoder is used to reconstruct the traces contained in the validation set. The validation set provides an unbiased evaluation of the model fit on the training set (validation loss). The training and validation losses decrease with the number of epochs (i.e., training events) and they both converge to $10^{-3}$ in 80 epochs. After training, for each $\mathcal{D}^{y}$, the encoder computes a set of 2-dimensional features $\mathcal{F}_{\mathrm{UAE}}^{y}=\left\{\mathbf{h}_{1}^{\mathrm{UAE}}, \ldots, \mathbf{h}_{n}^{\mathrm{UAE}}\right\}$.

\section{Clustering and Result Discussion}

We define the Centroid of the city $y$ as the centroid of the features of the city $y$, computed as:

$$
\mathbf{c}_{\mathrm{PCA}, \mathrm{UAE}}^{y}=\frac{\sum_{i=1}^{n} \mathbf{h}_{i}^{\mathrm{PCA}, \mathrm{UAE}}}{n}
$$




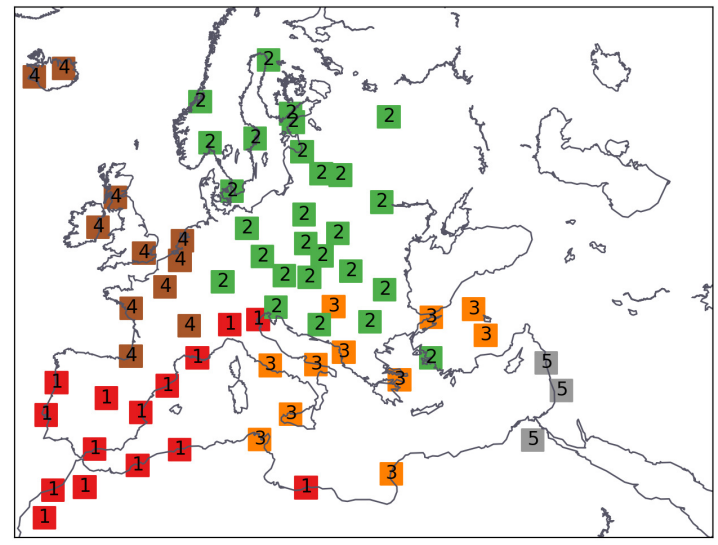

Fig. 4. Clusters obtained by using the UAE approach.

where the value of the centroid depends on the approach used to extract the features (i.e., PCA or UAE).

A distance-based clustering named agglomerative hierarchical clustering algorithm [16] is applied to the centroids to group the cities according to their similarities. The similarity between cities is expressed as the distance between their centroids.

The number of clusters has been selected by performing the silhouette analysis [17]. This technique provides a measure of similarity of an object in its own cluster compared to other clusters. The silhouette value ranges in the interval $[-1,1]$, being the highest value the best match of the object in the cluster and the smallest the poorest. The configuration is appropriate if most of the objects have a high value. Otherwise, the cluster configuration may have too many or too few classes. We have set the clustering algorithm to find the number of clusters that maximize the average silhouette value and we obtained 2 clusters in the case of PCA and 5 clusters in the case of UAE.

The result of the clustering based on PCA feature centroids is shown in Fig. 3, whereas the map obtained using UAE is reported in Fig. 4. Moreover, we show the average hourly solar energy generated for each cluster obtained with PCA and UAE in Fig. 5 and Fig. 6, respectively. The analysis of such variable for each cluster drives our interpretation of the extracted features to a more understandable model. In fact, we can distinguish four different parameters characterizing each identified cluster, namely: average daily generated energy $E_{H}$, solar energy peak value $P_{v}$, solar energy peak hour $P_{h}$ and average daylight time $D$. The values of those parameters are reported in Table I and Table II for PCA and UAE, respectively.

The map obtained with PCA is divided into two clusters (Fig. 3). The first cluster covers the North-East area and is characterized by a low amount of energy generation (lower $E_{h}$ and $P_{v}$ ). The second cluster covers the South-West area and is characterized by higher solar
TABLE I

STATISTICS OF THE CLUSTERS OBTAINED ON FEATURES EXTRACTED WITH PCA

\begin{tabular}{c||cccc} 
Cluster & $P_{h}[\mathrm{hr}]$ & $P_{v}[\mathrm{kWh}]$ & $E_{h}[\mathrm{kWh}]$ & $D[\mathrm{hrs}]$ \\
\hline \hline 1 & 11 & 0.4 & 2.1 & 12 \\
2 & 12 & 0.6 & 3.2 & 12
\end{tabular}

TABLE II

STATISTICS OF THE CLUSTERS OBTAINED ON FEATURES EXTRACTED WITH UAE

\begin{tabular}{c||cccc} 
Cluster & $P_{h}[\mathrm{hr}]$ & $P_{v}[\mathrm{kWh}]$ & $E_{h}[\mathrm{kWh}]$ & $D[\mathrm{hrs}]$ \\
\hline \hline 1 & 12 & 0.6 & 3.1 & 12 \\
2 & 11 & 0.3 & 1.8 & 13 \\
3 & 11 & 0.5 & 2.5 & 11 \\
4 & 12 & 0.3 & 1.9 & 13 \\
5 & 10 & 0.6 & 2.9 & 11
\end{tabular}

energy generation. Note that the two clusters differ also for the peak hours $P_{h}$. On the other hand, from the map obtained by UAE (Fig. 4), we see that the North of Europe is divided into two parts (clusters 2 and 4). Those clusters have the same values of $P_{v}, D$, similar $E_{h}$, but they differ in terms of solar peak hour $P_{h}$. The south area is divided into three clusters (1,3 and 5). Those clusters are very similar in terms of peak energy value $P_{h}$, but they differ for peak hour $P_{h}$, average energy $E_{h}$ and daylight hours $D$.

Note that the parameter $E_{h}$ represents the variable used by common solar maps to discriminate the different locations. In fact, those maps are usually based on the average or annual amount of solar energy generation. Therefore, PCA and UAE are able to extract two and three new variables featuring the solar energy generation process, respectively. The difference on the number of extracted latent variables may be due to the non-linear activation functions of the autoencoder, which obtains a better projection of the input data into the feature space compared to the linear PCA.

The geographical representation described in this paper is based on the characterization of several different temporal behaviors of the solar energy generation in diverse geographical locations. In particular, we believe that it may be helpful in designing energy management systems that have to control industrial processes, which are strictly related to the human activity. In fact, the parameters identified by our analysis provide a direct relation between the solar energy generation process and the time of the day.

\section{Conclusions}

In this paper, we have proposed an unsupervised method to learn hidden features of the solar energy generation from a PV system that may give a more accurate characterization of the process. In a first step, solar radiation data has been converted into instantaneous solar power through a detailed source model. Then, 

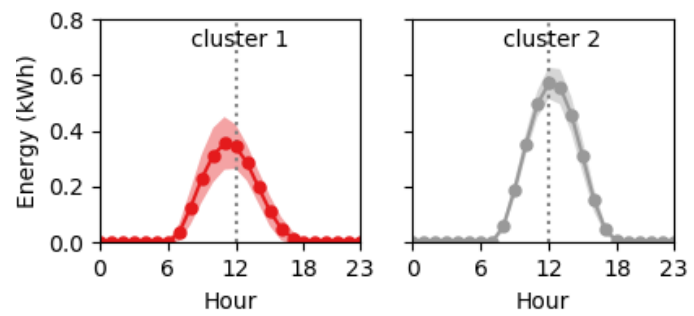

Fig. 5. Average hourly solar energy generation of the clusters obtained by using the PCA approach. The shaded area represents the standard deviation with respect to the other cities in the cluster.
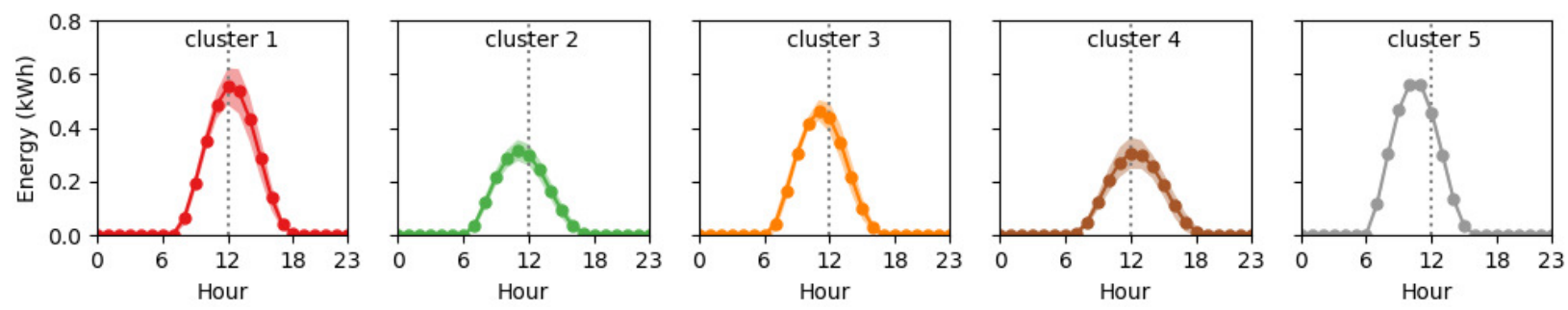

Fig. 6. Average hourly solar energy generation of the clusters obtained by using the UAE approach. The shaded area represents the standard deviation with respect to the other cities in the cluster.

two different approaches, namely PCA and autoencoder, have been used to extract meaningful features from the traces of the solar energy generation in an unsupervised manner. A hierarchical clustering algorithm has been used to group the locations according to their similarities in terms of solar energy generation. The results show that clustering on the extracted features provides support for learning latent variables of the solar energy generation process that may be used for a more detailed characterization of different geographical locations.

\section{ACKNOWLEDGMENT}

This work has received funding from the European Union Horizon 2020 research and innovation programme under the Marie Sklodowska-Curie grant agreement No 675891 (SCAVENGE) and by the Spanish Government under project TEC2017-88373-R (5GREFINE).

\section{REFERENCES}

[1] M. P. Mills, "The cloud begins with coal: Big data, big networks, big infrastructure, and big power," Digital Power Group, August 2013.

[2] A. S. Andrae and T. Edler, "On global electricity usage of communication technology: trends to 2030," MDPI Challenges, vol. 6, no. 1, pp. 117-157, April 2015.

[3] T. Han and N. Ansari, "Powering mobile networks with green energy," IEEE Wireless Communications, vol. 21, no. 1, pp. 9096, February 2014

[4] G. Piro, M. Miozzo, G. Forte, N. Baldo, L. A. Grieco, G. Boggia, and P. Dini, "HetNets Powered by Renewable Energy Sources: Sustainable Next-Generation Cellular Networks," IEEE Internet Computing, vol. 17, no. 1, pp. 32-39, January 2013.

[5] N. Piovesan, A. F. Gambin, M. Miozzo, M. Rossi, and P. Dini, "Energy sustainable paradigms and methods for future mobile networks: A survey," Computer Communications, vol. 119, pp. $101-117,2018$.
[6] M. Miozzo, D. Zordan, P. Dini, and M. Rossi, "Solarstat: Modeling photovoltaic sources through stochastic markov processes," in Energy Conference (ENERGYCON), 2014 IEEE International. IEEE, 2014, pp. 688-695.

[7] M. ri, T. A. Huld, E. D. Dunlop, and H. A. Ossenbrink, "Potential of solar electricity generation in the european union member states and candidate countries," Solar Energy, vol. 81, no. 10, pp. 1295 - 1305, 2007.

[8] Y. Bengio, A. Courville, and P. Vincent, "Representation learning: A review and new perspectives," IEEE Transactions on Pattern Analysis and Machine Intelligence, vol. 35, no. 8, pp. 1798-1828, Aug 2013.

[9] M. Freitag, S. Amiriparian, S. Pugachevskiy, N. Cummins, and B. Schuller, "audeep: Unsupervised learning of representations from audio with deep recurrent neural networks," arXiv preprint arXiv:1712.04382, 2017.

[10] A. K. Yadav and S. Chandel, "Solar radiation prediction using artificial neural network techniques: A review," Renewable and Sustainable Energy Reviews, vol. 33, pp. 772 - 781, 2014.

[11] SoDa. Solar radiation data. [Online]. Available: http://www.soda$\mathrm{pro}$.com/web-services/radiation/helioclim-3-archives-for-free

[12] N. Piovesan and P. Dini, "Optimal direct load control of renewable powered small cells: A shortest path approach," Internet Technology Letters, vol. 1, no. 1, p. e7.

[13] J. V. Dave, P. Halpern, and H. J. Myers, "Computation of incident solar energy," IBM Journal of Research and Development, vol. 19, no. 6, pp. 539-549, Nov 1975.

[14] I. T. Jolliffe, Principal components in regression analysis. Springer, 2002.

[15] I. Goodfellow, Y. Bengio, and A. Courville, Deep Learning. MIT Press, 2016.

[16] B. S. Everitt, S. Landau, M. Leese, and D. Stahl, Hierarchical Clustering. Wiley-Blackwell, 2011, ch. 4, pp. 71-110.

[17] P. J. Rousseeuw, "Silhouettes: a graphical aid to the interpretation and validation of cluster analysis," Journal of computational and applied mathematics, vol. 20, pp. 53-65, 1987. 